Research Paper

\title{
Eribulin Mesylate in Pretreated Breast Cancer Patients: A Multicenter Retrospective Observational Study
}

\author{
1. Medical Oncology Unit ASL Frosinone, Frosinone, Italy; \\ 2. Oncology Unit I, Azienda Ospedaliera Universitaria Pisana, Pisa, Italy; \\ 3. Division of Medical Oncology B, Regina Elena National Cancer Institute, Rome, Italy; \\ 4. Biostatistics Unit, Regina Elena National Cancer Institute, Rome, Italy; \\ 5. Division of Medical Oncology A, Regina Elena National Cancer Institute, Rome, Italy; \\ 6. Department of Medical Oncology, University Campus Bio-Medico, Rome, Italy; \\ 7. Oncology Unit, Sant'Andrea Hospital, Sapienza University of Rome, Rome, Italy; \\ 8. Medical Oncology, S.M. Goretti Hospital, Latina, Italy; \\ 9. Medical Oncology, San Pietro Hospital, Rome, Italy; \\ 10. Division of Medical Oncology, Department of Oncology, Belcolle Hospital, ASL Viterbo, Viterbo, Italy. \\ * These two authors contributed equally to this work and serve as first co-authors.
}

Teresa Gamucci ${ }^{*}$, Andrea Michelotti2 ${ }^{*}$, Laura Pizzuti ${ }^{3}$, Lucia Mentuccia ${ }^{1}$, Elisabetta Landucci², Isabella Sperduti ${ }^{4}$, Luigi Di Lauro ${ }^{3}$, Alessandra Fabi ${ }^{5}$, Giuseppe Tonini ${ }^{6}$, Valentina Sini ${ }^{7}$, Nello Salesi ${ }^{8}$, Ilaria Ferrarini $^{2}$, Angela Vaccaro ${ }^{1}$, Ida Pavese ${ }^{9}$, Enzo Veltri ${ }^{8}$, Luca Moscetti ${ }^{10}$, Paolo Marchetti ${ }^{7}$ and Patrizia Vici ${ }^{3}$

$\triangle$ Corresponding author: Patrizia Vici, MD. Division of Medical Oncology B, Regina Elena National Cancer Institute, Via Elio Chianesi 53, 00144 Rome (Italy). e-mail: pvici@ifo.it. Phone: 00390652665584 fax:00390652665075.

(c) Ivyspring International Publisher. This is an open-access article distributed under the terms of the Creative Commons License (http://creativecommons.org/ licenses/by-nc-nd/3.0/). Reproduction is permitted for personal, noncommercial use, provided that the article is in whole, unmodified, and properly cited.

Received: 2014.02.03; Accepted: 2014.02.26; Published: 2014.03.20

\begin{abstract}
Background: Eribulin was recently approved in patients progressing after being treated with anthracyclines and taxanes and after two or more chemotherapy lines for advanced disease.

Objectives: This multicenter observational retrospective study was performed in order to evaluate activity and tolerability of eribulin in real-world patient population.

Methods: 133 advanced breast cancer patients pretreated with $\geq 2$ chemotherapy lines for metastatic disease were retrospectively enrolled in the observational trial in II italian cancer centres.

Results: A median of 5 cycles of eribulin (range, I-15) were administered. Twenty-eight partial responses were observed, for an overall response rate of $21.1 \%(95 \% \mathrm{Cl}, 14 . \mathrm{I}-28.0)$. A stable disease was recorded in 57 patients $(42.8 \%$ ), and a clinical benefit (response or stable disease lasting $\geq$ six months) was observed in 5 I patients $(38.3 \%, 95 \% \mathrm{Cl}, 30 . \mathrm{I}-46.6)$. The subgroup analysis showed that a significant improvement in term of partial response and clinical benefit was achieved when eribulin was administered in HER-2 negative tumors ( $p=0.01$ and $p=0.004$, respectively) and when it is given as third-line $(p=0.09$ and $p=0.02$, respectively). Toxicity was manageable; fatigue is the most common side effect observed, usually of low-grade, and clearly cumulative-dose related.

Conclusions: In this retrospective, observational analysis eribulin confirmed its efficacy and manageable tolerability even in real-world population and in heavily pretreated patients.
\end{abstract}

Key words: advanced breast cancer, eribulin mesylate, real-world population, heavily pretreated patients, chemotherapy.

\section{Introduction}

There is a great need for treatments to improve the results in advanced and pretreated breast cancer patients, particularly when major classes of antineoplastic drugs such as anthracyclines and taxanes have 
been employed as adjuvant and/or first line treatment, and vinca alkaloids, gemcitabine, or capecitabine are the mainstay of treatment in this setting even if treatment guidelines have not deemed any particular chemotherapeutic regimen or single agent superior for second or further-line treatment $(1,2)$.

Eribulin mesylate is a structurally simplified synthetic analogue of halichondrin B (a natural product isolated from the marine sponge Halichondria okadai). Eribulin mechanism of action is different from other tubulin-targeting agents such as taxanes, vinca alkaloids, epothilones. It inhibits the growth phase of microtubule dynamics and sequesters tubulin into non-productive aggregates, inhibiting microtubule polymerization without affecting depolymerization, and inducing irreversible mitotic block at G2-M phases and apoptosis (3-5). The spectrum of action of eribulin is similar to that of vinca alkaloids. The drug is active, with a predictable side-effect profile in patients with extensively pretreated breast cancer. Three major phase II trials evaluated efficacy and safety of eribulin in advanced breast cancer patients, showing encouraging results in terms of activity and tolerability (6-8). A randomized phase III trial (EMBRACE) demonstrated overall survival advantage of eribulin compared to treatment of physician's choice in patients with heavily pretreated breast cancer, with manageable toxicity (9).

Eribulin was recently approved for metastatic breast cancer patients previously treated with at least 2 chemotherapeutic regimens for advanced disease, and previous therapy should include an anthracycline and a taxane in either adjuvant or metastatic setting. EMBRACE study is the only one trial in heavily pretreated breast cancer showing an advantage in overall survival, and having accrued a population reflecting what is actually seen in daily clinical practice. Eribulin is now being widely employed outside of clinical trials in Italy, as third or further line treatment.

On this basis, a multicenter observational retrospective study was undertaken in advanced breast cancer patients pretreated with $\geq 2$ chemotherapy lines for metastatic disease, in order to evaluate activity and tolerability of eribulin in real-world patient population.

\section{Patients and Methods}

Our analysis comprises advanced or metastatic breast cancer patients, all pretreated with anthracyclines and taxanes, and with previous two or more chemotherapy lines for advanced disease. Patients treated with eribulin in 11 Italian cancer centres were retrospectively enrolled in the observational trial. They all had an Eastern Cooperative Oncology Group (ECOG) of 2 or less, a life expectancy of more than 12 weeks, and adequate organs and haematological functions. Treatment schedule was like the registrative trial, eribulin $1.4 \mathrm{mg} / \mathrm{m} 2$ in 2-5 minutes intravenous on days 1 and 8 , on a three-weekly schedule, until disease progression, severe toxicity or patient refusal. Adverse events were assessed according to National Cancer Institute Common Terminology Criteria for Adverse Events (NCI-CTCAE, version 4), and treatment efficacy was evaluated by conventional RECIST criteria every three weeks or whenever clinically indicated. All patients provided a written informed consent, and institutional ethic committees approved the retrospective analysis.

\section{Statistical analysis}

A retrospective review of clinical and treatment data for all the patients was carried out, and data were entered on an anonymized database for data collection.

The standard summary statistics was used for both continuous and discrete variables. The objective response rate was reported with its $95 \%$ confidence interval. The associations were analyzed by the Chi-square test or Fisher exact test, when appropriate. The level of significance was set at $p \leq 0.05$. Overall survival (OS) and Progression-Free Suvival (PFS) were calculated by the Kaplan-Meier product-limit method. PFS was calculated as the time from the start date of therapy with eribulin to the date of progression or the date of the last follow-up evaluation. OS was calculated as the time from the start date of therapy with eribulin to the date of death or last contact. SPSS software was used for all statistical evaluations (SPSS version 21.0, SPSS Inc., Chicago, Illinois, USA).

\section{Results}

From March 2012 to August 2013, 133 advanced and pretreated breast cancer patients were treated in 11 Italian cancer centres. The study population comprises all patients who had received at least one eribulin cycle. Main patient and tumor characteristics are reported in Table 1 . Median age was 62 years, median ECOG performance status (PS) 1, 112 (84\%) patients had estrogen receptor (ER) and/or progesterone receptor (PgR) positive primary tumors, human epidermal growth factor 2 (HER2) was overexpressed or amplified in $28(21.1 \%)$ primary tumors; a triple negative subtype was recorded in 14 patients $(10.5 \%)$. The majority of the enrolled patients had visceral disease $(80.5 \%$ ), and more than $80 \%$ of the patients had multiple metastatic sites, with a median number of 2 sites. Seventy-seven percent of the patients overall, and $83 \%$ of the patients with hormonal receptors positive tumors had received adjuvant en- 
docrine treatment. Seventy percent of the patients, and $90 \%$ of the patients with hormonal receptor positive tumors had received one or more (up to 5) endocrine treatment for advanced disease, with a median number of 2 . Fifteen percent and $47.5 \%$ of the patients had received neoadjuvant/adjuvant chemotherapy, respectively. In regards to previous chemotherapy for advanced disease, $50 \%$ of the patients had been treated with 2 previous lines, and the remaining 50\% with more than 2 previous chemotherapy lines, up to 10 lines, not including the adjuvant setting. All patients had received previous anthracycline and taxane treatment, $59.4 \%$ and $89.5 \%$ of the patients as treatment for advanced disease, respectively. Ninety-eight patients $(73.7 \%)$ had also received previous capecitabine.

Table I. Main patient and tumor characteristics $(\mathrm{N}=\mid 33)$.

\begin{tabular}{|c|c|c|}
\hline Characteristic & $\mathrm{N}$ & $\%$ \\
\hline Median age, years (range) & \multicolumn{2}{|c|}{$62(30-79)$} \\
\hline Median ECOG PS (range) & \multicolumn{2}{|l|}{$1(0-2)$} \\
\hline \multicolumn{3}{|l|}{ ER/PgR status } \\
\hline ER and/or PgR + & 112 & 84 \\
\hline HER2 overexpressed/amplified & 28 & 21.1 \\
\hline Triple negative & 14 & 10.5 \\
\hline Prior neoadjuvant chemotherapy & 20 & 15 \\
\hline Prior adjuvant chemotherapy & 63 & 47.3 \\
\hline $\begin{array}{l}\text { Median prior lines of chemotherapy for advanced } \\
\text { disease (range) }\end{array}$ & $2(2-10)$ & \\
\hline \multicolumn{3}{|l|}{$\begin{array}{l}\text { Number of prior chemotherapy for advanced dis- } \\
\text { ease }\end{array}$} \\
\hline 2 & 66 & 49.7 \\
\hline 3 & 26 & 19.5 \\
\hline 4 & 15 & 11.3 \\
\hline$\geq 5$ & 26 & 19.5 \\
\hline \multicolumn{3}{|l|}{ Previous chemotherapy for advanced disease } \\
\hline Anthracyclines & 79 & 59.4 \\
\hline Taxanes & 119 & 89.5 \\
\hline Capecitabine & 98 & 73.7 \\
\hline Prior adjuvant hormonal therapy & 102 & 76.7 \\
\hline Prior hormonal therapy for advanced disease & 92 & 69.2 \\
\hline \multicolumn{3}{|l|}{ Dominant disease site } \\
\hline Viscera & 107 & 80.5 \\
\hline Bone & 8 & 6 \\
\hline Soft tissue & 18 & 13.5 \\
\hline \multicolumn{3}{|l|}{ Number of disease site } \\
\hline 1 & 23 & 17.3 \\
\hline 2 & 53 & 39.8 \\
\hline 3 & 38 & 28.6 \\
\hline$\geq 4$ & 19 & 14.6 \\
\hline Eribulin cycles administered, median (range) & $5(1-15)$ & \\
\hline
\end{tabular}

In the present retrospective analysis patients received a median of 5 cycles of eribulin (range, 1-15).

Regarding toxicity (Table 2), 89 patients $(66.9 \%)$ experienced asthenia/fatigue, which was mild to moderate in $63.9 \%$ of the cases, being of grade 3 in only $3 \%$ of the patients. The symptom was clearly cumulative dose-related, appearing usually after a median of 4 cycles. Peripheral neurotoxicity of any grade was observed in $35.3 \%$ of the patients, being of grade 1 in $18 \%$ and of grade 2 in $13.5 \%$ of the patients; neurotoxicity of grade 3 was observed in 5 patients $(3.8 \%)$. No grade 4 neurotoxicity was recorded. The symptom was cumulative-dose related, occurring usually after the fourth cycle, with significant differences in incidence and severity depending on the number of cycles administered ( $\geq 6$ cycles: $p$ 0.0001), and rapidly improving or disappearing after a dose-reduction of 6-8 weeks. Treatment discontinuation depending on neurotoxicity was recorded in 3\% of the patients. Severe (G3) mucositis was rarely observed $(1.5 \%)$, being usually of grade $1-2(23.3 \%)$. Alopecia was encountered in $40 \%$ of the patients. Gastrointestinal toxicity, mainly nausea and vomiting or diarrhea was observed in $28.8 \%$ of the patients, being usually mild to moderate. We do not have details on single gastrointestinal toxicity. Constipation was rarely observed (8 patients), usually of grade 1 and short-lasting. Twenty patients experienced mild arthralgias. We did not observe any cutaneous toxicity.

Table 2. Main toxicity in 133 patients according to National Cancer Institute Common Terminology Criteria version 4.0.

\begin{tabular}{|c|c|c|c|}
\hline Toxicity & Grade $1 \mathrm{~N}(\%)$ & Grade $2 \mathrm{~N}(\%)$ & Grade $3 \mathrm{~N}(\%)$ \\
\hline Neutropenia & $11(8.3)$ & $8(6)$ & $19(14.3)$ \\
\hline Thrombocytopenia & $5(3.8)$ & $3(2.3)$ & $3(2.3)$ \\
\hline Anemia & $11(8.3)$ & $7(5.2)$ & $1(0.8)$ \\
\hline Hypertransaminasemia & $13(9.7)$ & $5(3.8)$ & $1(0.8)$ \\
\hline Fatigue & $50(37.6)$ & $35(26.3)$ & $4(3)$ \\
\hline Neurotoxicity & $24(18)$ & $18(13.5)$ & $5(3.8)$ \\
\hline Mucositis & $18(13.5)$ & $13(9.8)$ & $2(1.5)$ \\
\hline Gastrointestinal toxicity & $24(18)$ & $13(9.8)$ & $1(0.8)$ \\
\hline Arthralgias & $17(12.7)$ & $3(2.2)$ & - \\
\hline Alopecia* & $37(27.8)$ & $18(13.5)$ & - \\
\hline
\end{tabular}

*For alopecia only grade 1-2 are indicated.

Neutropenia was of grade 1-2 and of grade 3 in $14.3 \%$ of the patients, respectively; no grade 4 neutropenia was encountered. No data on febrile neutropenia incidence are available. Thrombocytopenia was occasional, $13.5 \%$ of the patients developed mild anemia or transient transaminases increase, respectively. In $47(35.3 \%)$ patients granulocyte colony stimulating factor (G-CSF) was administered, according to local practice. A $25 \%$ drug dose reduction was performed in $33.8 \%$ of the patients, mostly depending on neurotoxicity, asthenia, haematological toxicity. No data on treatment delays were available.

Toxicity-related eribulin discontinuation was performed in 6 patients (4.5\%), depending on neurotoxicity ( 3 patients) and asthenia ( 3 patients). 
No significant differences in toxicity were observed according to age, and even in the 28 patients older than 70 years treatment was well tolerated; the only difference was that G3 asthenia was more frequently observed $(10.7 \%$ versus $1.0 \%)$, but numbers were very small.

In regards to efficacy all but one patient were evaluable for response. We did not observe complete responses, 28 partial responses in 133 patients recruited were recorded, for an overall response rate of 21.1\% (95\%CI,14.1-28.0). A stable disease was observed in 57 patients (42.8\%). Clinical benefit, defined as response or stable disease lasting $\geq$ six months, was observed in 51 patients (38.3\%; 95\% CI, 30.1-46.6). Objective responses were observed in all disease sites, being $18.9 \%$ in visceral, $50 \%$ in bone, $22.2 \%$ in soft tissue lesions (Table 3 ).

Table 3. Objective response in 133 enrolled patients.

\begin{tabular}{lll}
\hline Responses & No. of patients & \% (C.I. 95\%) $^{\text {95\% }}$ \\
\hline Partial response & 28 & 21.1 (14.1-28.0) \\
Stable disease & 57 & 42.8 \\
Progressive disease & 47 & 35.3 \\
Clinical benefit ${ }^{\mathrm{b}}$ & 51 & 38.3 \\
\hline
\end{tabular}

${ }^{a}$ C.I. Confidence interval ${ }^{b}$ Response or stable disease lasting $\geq 6$ months.

Treatment activities by different tumor subtypes and according to treatment line were reported on Table 4 . Responses by triple negative or no triple negative subtype were identical $(21.4 \%)$, but numbers were small. Only one response was reported in HER2 positive tumors $(3.6 \%)$, whereas $25(25 \%)$ objective responses were observed in HER2 negative tumors $(p=0.01)$. We observed 25 responses in hormonal receptor positive tumors $(22.5 \%)$ and 3 responses were observed in receptor negative tumors $(14.3 \%)$; the difference was not statistically significant (p 0.56). In regards to number of metastatic sites $41 \%$ of response rate was observed in one only metastatic site, whereas in 19 patients with more than one site response rate was $17.3 \%(p=0.01)$. One brain objective response was observed among 12 patients with known brain metastases. Responses related to previous chemotherapy lines were as follows: $27.3 \%$ in patients treated as third-line for advanced disease, $15.2 \%$ in patients treated beyond the third-line $(\mathrm{p}=0.09)$.

No significant differences in clinical benefit (Table 5) was observed between triple negative and no triple negative tumors $(35.7 \%$ vs $38.5 \%)$, or according to disease sites $(p=0.09)$, whereas a statistically significant difference in favour of HER2 negative tumors was observed $(p=0.004)$. Clinical benefit was observed in $28.6 \%$ of patients with hormonal receptor negative tumors, and in $40.5 \%$ patients with positive hormonal receptor, without any significant differences. Clinical

benefit was more frequently observed when eribulin was administered as third-line (48.5\%), in comparison with more advanced lines $(28.8 \%)(\mathrm{p}=0.02)$, and in one only metastatic site $(\mathrm{p}=0.03)$, in comparison with multiple sites.

Table 4. Response by tumor subtypes and treatment-line.

\begin{tabular}{lll}
\hline Characteristic & $\begin{array}{l}\text { Partial response } \\
\text { N }(\%)\end{array}$ & $p$ \\
\hline ER/PgR status & $25(22.5)$ & 0.56 \\
ER and/or PgR positive & $3(14.3)$ & \\
ER and/or PgR negative & & \\
Triple negative & $3(21.4)$ & 0.99 \\
Yes & $25(21.4)$ & \\
Not & & \\
HER2 & $1(3.6)$ & 0.01 \\
Overexpressed/amplified & $25(25.0)$ & \\
Not overexpressed/amplified & & \\
Dominant disease site & $20(18.9)$ & 0.11 \\
Viscera & $4(50.0)$ & \\
Bone & $4(22.2)$ & \\
Soft tissue & & \\
Brain metastases & $1(8.3)$ & \\
Yes & $27(22.5)$ & \\
Not & & \\
Number of disease site & $9(41)$ & \\
1 & $19(17.3)$ & \\
$\geq 2$ & & \\
Number of prior chemotherapy for advanced & & \\
disease & $18(27.3)$ & 0.01 \\
2 & $10(15.2)$ & \\
$\geq 3$ & & \\
\hline ER: estrogen receptor; PgR: progesterone receptor; HER2: human epidermal \\
growth factor 2.
\end{tabular}

Table 5. Clinical benefit.

\begin{tabular}{lll}
\hline Characteristic & $\begin{array}{l}\text { Clinical benefit, N } \\
(\%)\end{array}$ & $p$ \\
\hline ER/PgR status & $45(40.5)$ & \\
ER and/or PgR positive & $6(28.6)$ & 0.3 \\
ER and/or PgR negative & $5(35.7)$ & 0.84 \\
Triple negative tumors & $45(38.5)$ & \\
Yes & & \\
Not & $4(14.3)$ & 0.00 \\
HER2 & $44(44.0)$ & 4 \\
Overexpressed/amplified & & \\
Not overexpressed/amplified & $38(35.8)$ & \\
Dominant disease site & $6(75.0)$ & 0.09 \\
Viscera & $7(38.9)$ & \\
Bone & & \\
Soft tissue & $2(16.7)$ & \\
Brain metastases & $49(40.8)$ & \\
Yes & & \\
Not & $13(59.1)$ & \\
Number of disease site & $38(34.5)$ & \\
1 & & \\
$\geq 2$ & $32(48.5)$ & \\
Number of prior chemotherapy for ad- & $19(28.8)$ & \\
vanced disease & & \\
2 & &
\end{tabular}


At the time of the analysis, $22.7 \%$ of the patients were still in response or stability of disease, and $66.7 \%$ of the patients were still alive. At a median follow up of 8.3 months (range, 1-19), the median response duration was 5.2 months $(95 \% \mathrm{CI}, 4.2-6.3)$, the median progression free survival was 4.4 months $(95 \% \mathrm{CI}$, 3.7-5), and the median overall survival 14.3 months (95\% CI, 11.7-16.8) (Figure 1). One-year progression free survival was $9.7 \%$, and 1-year overall survival $58 \%$.
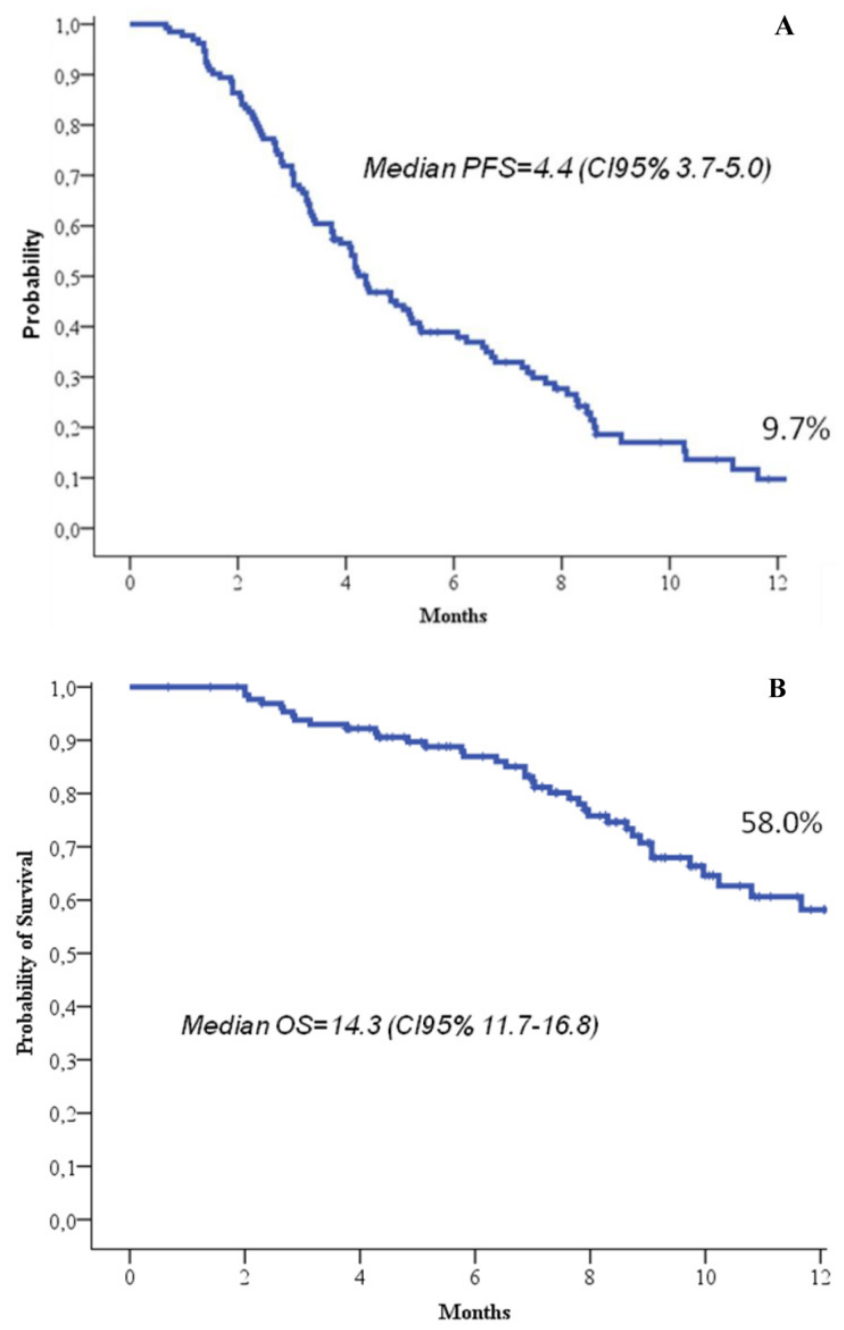

Figure I. Progression free survival (a) and overall survival (b).

\section{Discussion}

Despite advances in terms of efficacy and tolerability of treatments have been achieved over the last two decades, the prognosis of advanced breast cancer patients remains poor, and the goals for the vast majority of the patients, unfortunately, are not to find a cure, but to obtain disease control and symptoms palliations, possibly maintaining an acceptable quality of life. The benefit in overall survival also constitutes a relevant goal, but usually multiple chemotherapy lines and cross-over designs render difficult to value this outcome (10), even if more recent data from population-based studies and clinical trials show an improvement, with a median overall survival increasing from 18 to 24 months in the last years (11-13). In contrast to the adjuvant setting, where multi-agent chemotherapy regimens containing anthracyclines and/or taxanes are standard, the single-agent therapy approach is preferred in metastatic disease, unless rapid disease control is required in view of pending visceral crisis.

Until now, treatment guidelines did not clearly identify a specific regimen or single agent after the first-line in advanced setting, treatment choice depending on disease burden, tumor subtype, prior therapeutic exposure, and the use of multiple lines of treatment is still under evaluation, particularly after the second-line. At present, there is various supports for the use of third-line chemotherapy, and even beyond the third-line recent retrospective studies suggest a potential gain, since each line can contribute to longer survival (14-16). The heterogeneity of the disease and the variability in individual presentations means that no widely accepted specific treatment guidelines exist.

Even if no standard third-line treatment for advanced disease is still clearly established, results from the EMBRACE trial are very encouraging, since a significant advantage in survival was not previously reported with a single agent in advanced setting. In addition it is well known that many patients with heavily pretreated breast cancer may benefit from later lines of therapy $(17,18)$, thus the availability of an active and well tolerated agent such as eribulin is of particular relevance (19). Moreover, EMBRACE trial accrued a population cohort very heavily pretreated, which reflects what is realistically seen in daily clinical practice.

In fact, the lack of reproducibility in real world-population of the results of many registrative trials in oncology is an increasing concern, and there are high rates of failure in validating and confirming promising results (20). Among the multiple reasons why an early result cannot be confirmed in clinical practice are that patients enrolled in clinical trials are selected, and patients in real-world are usually more heterogeneous, with comorbidities or borderline organ functions, and with lower treatment compliance. From this point of view, observational retrospective studies like the present study are considered good alternative sources of information for treatments (21).

Our retrospective analysis on eribulin use in advanced/metastatic pretreated breast cancer confirms in fact that, even in real-world population, the treatment maintained favourable outcomes, in terms 
of response rate, clinical benefit, progression free survival and overall survival, with a manageable profile of toxicity, not significantly different from the registrative trial. The results of the present analysis are particularly encouraging, given that we observed an overall response rate of $21.1 \%$, a clinical benefit of $38.6 \%$, a median progression free survival of 4.4 months and a median overall survival of 14.3 months which all favourably compares with the registrative trial, and in a patient population with a similar pre-treatment amount to the EMBRACE trial.

Due to the relatively small number of patients in the present analysis, we cannot suggest treatment efficacy by tumor subgroups, even if activity was observed in all metastatic disease sites and tumor subtypes. In particular, we cannot confirm EMBRACE results on eribulin efficacy in triple negative subtype, due to the small sample size. In regards to hormonal receptor status, no significant differences were observed in the two subgroups, and eribulin appeared active even in ER positive tumors. A higher eribulin efficacy was observed in Her-2 negative tumors.

Adverse events in the present study did not significantly differ from the EMBRACE trial, confirming asthenia/fatigue as the most common side effect observed, occurring in $70.1 \%$ of the patients, slightly more common than in the EMBRACE trial, but usually of low-grade, and clearly cumulative-dose related. Peripheral neurotoxicity was recorded in $35.3 \%$ of the patients, being severe (grade 3 ) in only $3.8 \%$ of the patients, which favourably compares with the registrative trial, where these issues were $35 \%$ and $8 \%$. The symptom was cumulative-dose related and rapidly improving or disappearing after transient dose-reduction or discontinuation of the drug. We report a comparable amount of gastrointestinal toxicity and alopecia, and a slightly higher incidence of mucositis, even if it was usually mild. In regards to haematological toxicity we observed less neutropenia and anemia, but more patients in our casistic received G-CSF support compared to the registrative trial. Eribulin dose-reduction depending on toxicity was rare, being only $4.5 \%$.

Our analysis confirm that an early use of eribulin (after the second-line of chemotherapy for advanced setting) is preferred to the use as subsequent lines in order to obtain more favourable results in terms of efficacy $(27.3 \%$ response and $48.5 \%$ clinical benefit rates, respectively), even if the treatment maintained activity also in subsequent lines $(15.2 \%$ and $28.8 \%$ response and clinical benefit rates, respectively). Moreover, responses and clinical benefits were observed in very heavily pre-treated patients as well.

Various other agents, including gemcitabine, vinorelbine, capecitabine, nab-paclitaxel, and ixa- bepilone have been studied and employed in clinical practice for the treatment of advanced lines in metastatic breast cancer, however, even if these agents may be considered potential alternatives, none of them may be considered the standard of care (10). Data on single agent gemcitabine in anthracycline and taxane pre-treated patients suggest an overall response rate ranging from $12 \%$ to $30 \%$, but with significant haematological toxicity (10), where more favourable results have been observed in combination with taxanes (22-24). Among vinca alkaloids, vinorelbine is widely employed in pretreated breast cancer, in anthracycline and taxane pretreated patients yielding response rates ranging from $10 \%$ to $35 \%$, depending on the amount of the pre-treatment (25). It is usually well tolerated, but it is frequently employed in combination regimens, and no evidence of survival benefit from randomized trials are reported (26-28). Additional agents include nab-paclitaxel, which in phase II trials yielded a response rate of $15 \%$ in anthracycline and taxane pretreated patients (29), and ixabepilone, with an encouraging efficacy profile, but usually yielding higher incidence of neurotoxicity, up to $63 \%$ of the patients (30-32). Among the most commonly employed drugs in second and third chemotherapy line is capecitabine, an antimetabolite agent approved for treating patients after failure of anthracycline and taxane treatment. A review of 28 single-agent capecitabine trials reported a median overall response rate of $28 \%$, with a median overall survival of 11 months, and a good tolerability, but in patients less heavily pretreated than those enrolled in the EMBRACE trial (33). A recent pooled analysis of data from capecitabine monotherapy clinical trials showed in second-line a response rate of $19 \%$, a progression free survival of 3.7 months, and an overall survival of 13 months, with safety comparable to previous studies (34).

Preliminary results of a phase III randomized trial comparing capecitabine with eribulin as first, second and third-line showed a non-significant trend (HR 0.88, p 0.056) in overall survival in favour of eribulin. It suggested, at a pre-planned exploratory analysis, a greater therapeutic eribulin benefit in triple negative, ER and HER2 negative subgroups (35), in a patient population less heavily pretreated than in the EMBRACE trial or in the present retrospective analysis.

Overall, none of the above mentioned single agents truly showed any benefit in overall survival over other treatments in very heavily pretreated patients, as reported in the EMBRACE trial, since agents or regimens which are able to prolong survival in patients treated beyond the second-line, as reported in some studies, include taxanes or unusual combinations (18), and, to our knowledge, eribulin is the only 
one agent showing survival advantage when used as third-line or beyond for metastatic disease.

The main weakness of the present analysis is the retrospective observational design: many items are not defined from a predefined study protocol, and there is complete data information lacking for some patients and/or characteristics; however, this is commonly observed in retrospective analyses.

The strength of this study is that it represents data from a cohort of patients outside of clinical trials, providing a realistic picture of what truly takes place in the daily population. In fact, one relevant limitation outside of clinical trials is that many patients do not fit the profile of clinical study participants, mainly in very advanced settings, where comorbidities or disease extension are relevant, and results are frequently disappointing, not reproducing clinical trial benefits.

To our knowledge, only two studies with small sample size reported in full text the use of eribulin in real-world population, with similar results $(36,37)$.

Indeed, waiting for the discovery of innovative biological therapies (38), the recent approval of eribulin after second-line treatment for advanced breast cancer expands the treatment options available for patients with late-stage disease and, in this setting; our study confirms favourable results in terms of activity and tolerability even in daily clinical practice where it may be considered of significant importance.

\section{Acknowledgements}

We thank Anna Maria Edlisca and Tania Merlino for technical assistance.

\section{Authors' contributions}

TG conceived and designed the study. TG, AM, LP, LM, EL, LDL, AF, GT, VS, NS, BS, AV, IP, EV, LM, $\mathrm{PM}$ and PV collected and assembled the data. IS performed the statistical analysis. PV wrote the manuscript. All authors read and approved the final manuscript.

\section{Competing Interests}

The authors have declared that no competing interest exists.

\section{References}

1. El Saghir NS, Tfayli A, Hatoum HA, Nachef Z, Dinh P, Awada A. Treatment of metastatic breast cancer: state-of-the-art, subtypes and perspectives. Crit Rev Oncol Hematol. 2011; 80: 433-49

2. Moreno-Aspitia A, Perez EA. Treatment options for breast cancer resistant to anthracycline and taxane. Mayo Clin Proc. 2009; 84: 533-45

3. Jordan MA, Kamath K, Manna T, et al. The primary antimitotic mechanism of action of the synthetic halichondrin E7389 is suppression of microtubule growth. Mol Cancer Ther. 2005; 4: 1086-95

4. Smith JA, Wilson L, Azarenko O, et al. Eribulin binds at microtubule ends to a single site on tubulin to suppress dynamic instability. Biochemistry. 2010; 49: 1331-7

5. Towle MJ, Salvato KA, Wels BF, et al. Eribulin induces irreversible mitotic blockade: implications of cell-based pharmacodynamics for in vivo efficacy under intermittent dosing conditions. Cancer Res. 2011; 71: 496-505
6. Cortes J, Vahdat L, Blum JL, et al. Phase II study of the halichondrin B analog eribulin mesylate in patients with locally advanced or metastatic breast cancer previously treated with an anthracycline, a taxane, and capecitabine. J Clin Oncol. 2010; 28: 3922-8.

7. Vahdat LT, Pruitt B, Fabian CJ, et al. Phase II study of eribulin mesylate, a halichondrin B analog, in patients with metastatic breast cancer previously treated with an anthracycline and a taxane. J Clin Oncol. 2009; 27: 2954-61.

8. Aogi K, Iwata H, Masuda N, et al. A phase II study of eribulin in Japanese patients with heavily pretreated metastatic breast cancer. Ann Oncol. 2012; 23: 1441-8.

9. Cortes J, O'Shaughnessy J, Loesch D, et al. Eribulin monotherapy versus treatment of physician's choice in patients with metastatic breast cancer (EMBRACE): a phase 3 open-label randomised study. Lancet. 2011; 377: 914-23.

10. Ayoub JP, Verma Sh, Verma S. Advances in the management of metastatic breast cancer: options beyond first-line chemotherapy. Curr Oncol. 2012; 19: 91-105.

11. Dawood S, Broglio K, Gonzalez-Angulo AM, Buzdar AU, Hortobagyi GN, Giordano SH. Trends in survival over the past two decades among white and black patients with newly diagnosed stage IV breast cancer. J Clin Oncol. 2008; 26: 4891-8.

12. Mauri D, Polyzos NP, Salanti G, Pavlidis N, Ioannidis JP. Multiple-treatments meta-analysis of chemotherapy and targeted therapies in advanced breast cancer. J Natl Cancer Inst. 2008; 100: 1780-91.

13. Gennari A, D'amico M, Corradengo D. Extending the duration of first-line chemotherapy in metastatic breast cancer: a perspective review. Ther Adv Med Oncol. 2011; 3: 229-32.

14. Tacca O, LeHeurteur M, Durando X, et al. Metastatic breast cancer: overall survival related to successive chemotherapies. What do we gain after the third line? Cancer Invest. 2009; 27: 81-5.

15. Bernardo G, Palumbo R, Poggi G, et al. Beyond the second line chemotherapy in metastatic breast cancer: when stop the treatment between science and conscience. Cancer Res. 2010; 70 (Suppl 24): 446s.

16. Planchat $\mathrm{E}, \mathrm{Abrial} \mathrm{C}$, Thivat $\mathrm{E}$, et al. Late lines of treatment benefit survival in metastatic breast cancer in current practice? Breast. 2011; 20: 574-8.

17. Dufresne A, Pivot X, Tournigand C, et al. Impact of chemotherapy beyond the first line in patients with metastatic breast cancer. Breast Cancer Res Treat. 2008; 107: 275-9.

18. Palumbo R, Sottotetti F, Riccardi A, et al. Which patients with metastatic breast cancer benefit from subsequent lines of treatment? An update for clinicians. Ther Adv Med Oncol. 2013; 5: 334-50.

19. Gradishar WJ. The place for eribulin in the treatment of metastatic breast cancer. Curr Oncol Rep. 2011; 13: 11-6.

20. Pusztai L, Hatzis C, Andre F. Reproducibility of research and preclinical validation: problems and solutions. Nat Rev Clin Oncol. 2013 Dec;10(12):720-4.

21. Korn EL, Freidlin B. Methodology for comparative effectiveness research: potential and limitations. J Clin Oncol. 2012; 30: 4185-7.

22. Vici P, Capomolla E, Foggi $\mathrm{P}$, et al. High activity of salvage treatment with biweekly paclitaxel-gemcitabine combination in heavily pretreated breast cancer patients. J Exp Clin Cancer Res. 2006; 25: 39-44.

23. Vici P, Fabi A, Metro G, et al. Phase I-II trial of prolonged gemcitabine infusion plus paclitaxel as a biweekly schedule for advanced breast cancer patients pretreated with anthracyclines. Cancer Chemother Pharmacol. 2011; 67: 687-93.

24. Albain KS, Nag SM, Calderillo-Ruiz G, et al. Gemcitabine plus Paclitaxel versus Paclitaxel monotherapy in patients with metastatic breast cancer and prior anthracycline treatment. J Clin Oncol. 2008; 26: 3950-7.

25. Pugliano L, de Azambuja E. Treatment options in anthracycline and/or taxane pretreated patients with metastatic breast cancer. Onkologie. 2012; 35: 476-8.

26. Vici P, Di Lauro L, Carpano S, et al. Vinorelbine and mitomycin C in anthracycline-pretreated patients with advanced breast cancer. Oncology. 1996; 53: 16-8.

27. Vici P, Di Lauro L, Sergi D, et al. A phase II trial of docetaxel and vinorelbine in patients with advanced breast cancer previously treated with anthracyclines. Oncology. 2008; 75: 175-81.

28. Valero V, Hortobagyi GN. Are anthracycline-taxane regimens the new standard of care in the treatment of metastatic breast cancer? J Clin Oncol. 2003; 21: 959-62.

29. Blum JL, Savin MA, Edelman G, et al. Phase II study of weekly albumin-bound paclitaxel for patients with metastatic breast cancer heavily pretreated with taxanes. Clin Breast Cancer. 2007; 7: 850-6.

30. Thomas E, Tabernero J, Fornier M, et al. Phase II clinical trial of ixabepilone (BMS-247550), an epothilone B analog, in patients with taxane-resistant metastatic breast cancer. J Clin Oncol. 2007; 25: 3399-406.

31. Perez EA, Lerzo G, Pivot X, et al. Efficacy and safety of ixabepilone (BMS-247550) in a phase II study of patients with advanced breast cancer resistant to an anthracycline, a taxane, and capecitabine. J Clin Oncol. 2007; 25: 3407-14.

32. Vahdat LT, Garcia AA, Vogel C, et al. Eribulin mesylate versus ixabepilone in patients with metastatic breast cancer: a randomized Phase II study comparing the incidence of peripheral neuropathy. Breast Cancer Res Treat. 2013; 140: 341-51.

33. Ershler WB. Capecitabine monotherapy: safe and effective treatment for metastatic breast cancer. Oncologist. 2006; 11: 325-35. 
34. Blum JL, Barrios $\mathrm{CH}$, Feldman N, et al. Pooled analysis of individual patient data from capecitabine monotherapy clinical trials in locally advanced or metastatic breast cancer. Breast Cancer Res Treat. 2012; 136: 777-88.

35. Kaufman PA, Awada A, Twelves C, et al. A phase III, open-label, randomized, multicenter study of eribulin mesylate versus capecitabine in patients with locally advanced or metastatic breast cancer previously treated with anthracyclines and taxanes. Presented at: 2012 CTRC-AACR San Antonio Breast Cancer Symposium; December 4-8, 2012; San Antonio, Texas. Abstract S6-6.

36. Poletti P, Ghilardi V, Livraghi L, Milesi L, Rota Caremoli E, Tondini C. Eribulin mesylate in heavily pretreated metastatic breast cancer patients: current practice in an Italian community hospital. Future Oncol. 2014; 10: 233-9.

37. Ramaswami R, O'Cathail SM, Brindley JH, Silcocks P, Mahmoud S, Palmieri C. Activity of eribulin mesylate in heavily pretreated breast cancer granted access via the Cancer Drugs Fund. Future Oncol. 2013; [Epub ahead of print].

38. Tinoco G, Warsch S, Glück S, Avancha K, Montero AJ. Treating breast cancer in the 21st century: emerging biological therapies. J Cancer. 2013; 4: 117-32. 